\title{
GPPS-TC-2019-0014
}

\section{Purge Flow Effects on Rotor Hub Endwall Heat Transfer with Extended Endwall Contouring into the Disk Cavity}

\author{
Dominic D. Hänni \\ Laboratory for Energy \\ Conversion, ETH Zurich \\ dohaenni@ethz.ch \\ Zurich, Switzerland
}

\author{
Rainer Schädler \\ Laboratory for Energy \\ Conversion, ETH Zurich \\ schaedler@lec.mavt.ethz.ch \\ Zurich, Switzerland
}

\author{
Reza S. Abhari \\ Laboratory for Energy \\ Conversion, ETH Zurich \\ rabhari@lec.mavt.ethz.ch \\ Zurich, Switzerland
}

\author{
Anestis I. Kalfas \\ Aristotle University of Thessaloniki \\ akalfas@auth.gr \\ Thessaloniki, Greece
}

\author{
Ewald Lutum \\ MTU Aero Engines AG \\ ewald.lutum@mtu.de \\ Munich, Germany
}

\author{
Gregor Schmid \\ Siemens AG \\ gregor.schmid@siemens.com \\ Muelheim an der Ruhr, Germany \\ Nicolas Lecoq \\ MTU Aero Engines AG \\ nicolas.lecoq@mtu.de \\ Munich, Germany
}

\section{ABSTRACT}

Efficiency improvements for gas turbines are strongly coupled with increasing turbine inlet temperatures. This imposes new challenges for designers for efficient and adequate cooling of turbine components. Modern gas turbines use bleed air from the compressor to inject into the stator/rotor rim seal cavity to prevent hot gas ingestion from the main flow, while cooling the rotor disk. This purge flow interacts with the main flow field and static pressure field imposed by the turbine blades. The complex interaction causes nonuniform and jet-like penetration of the purge flow into the main flow field and therefore affects the endwall heat transfer on the rotor.

In order to improve the understanding of purge flow effects on rotor hub endwall heat transfer, measurements in the 1.5-stage axial turbine facility LISA at ETH Zurich have been performed. An unshrouded, high-pressure representative turbine design with 3D blading and extended endwall contouring of the rotor into the cavity seal has been tested. A state-of-the-art measurement setup with a high-speed infrared camera and thermally managed rotor insert has been used to perform high-resolution heat transfer measurements on the rotor.

Three different purge flow rates were investigated with regard to hub endwall heat transfer. Additionally, steady-state CFD simulations were performed to complement the experiments. It was found that the local heat transfer rate changes up to $\pm 20 \%$ depending on the purge flow rate. The main part of the purged air is ejected at the endwall trough location and swept towards the rotor suction side caused by the interaction of main flow and the cavity extended endwall design. The presence of low momentum purge flow is locally reducing the heat transfer rate. Changes in adiabatic wall temperature and heat transfer depending on purge rate are observed from the platform start up to the cross passage migration of the secondary flow structures.

\section{INTRODUCTION}

Over the past years, various design approaches were developed to increase efficiency and reduce the losses due to secondary flows in turbomachines. Up to $30-50 \%$ of the total aerodynamic losses can be attributed to secondary flow losses according to (Sharma and Butler, 1987). (Langston, 2001) provides a condensed review about secondary flows in turbomachines. A promising approach to reduce and control the cross passage flow due to the pressure gradient is endwall contouring.

Studies by (Harvey et al., 1999) showed that the usage of non-axisymmetric endwall contouring is a powerful tool for reducing secondary flows with comparable effects to lean and skew of blades. Cascade measurements confirmed a reduction of secondary losses by $30 \%$ (Hartland et al., 1999). An efficiency increase of $1.0 \%$ and $0.4 \%$ is also reported by (Schuepbach et al., 2008) and (Snedden et al., 2009) respectively. 
For high pressure turbines, the presence of cavity leakage flows for cooling the rotor disk and prevent hot gas ingestion is omnipresent and needed. Measurements by (Jenny et al., 2013) showed that the presence of purge is increasing the unsteadiness and increases the rotor hub passage losses by $4 \%$ for each percent of purge flow injected. The beneficial effect of endwall contouring was also confirmed by (Regina et al., 2014). However, they found that endwall contouring increases the sensitivity of stage efficiency to purge flow injection and eventually the beneficial impact is lost. Similar results are reported by (Schuepbach et al., 2010), where a reduction of $1.2 \%$ in efficiency per percent of purge injection was found.

The effect of purge flow and endwall contouring on heat transfer is mainly investigated in linear cascades. (Thrift et al., 2012) showed that a slot angle of $45 \mathrm{deg}$ increases cooling effectiveness compared to $90 \mathrm{deg}$ due to a different formation of the horse shoe vortex. An overall averaged reduction in Nusselt number for the endwall with and without purge flow is reported by (Roy et al., 2017) for a contoured endwall due to secondary flow control. Also (Lynch et al., 2010) reported a reduction by $3.1 \%$ of averaged heat transfer for endwall contouring. However, local increase was observed around the blade leading edge region where the horse shoe vortex forms and a decrease at the suction side of the blade. The same authors reported that increased purge flow rates increase the heat transfer on the suction side due strengthening of the vortical flow structures. An area-averaged increase by $8 \%$ is reported for heat transfer and an increase of cooling effectiveness by $27 \%$.

Heat transfer measurements in rotating facilities are not widely available in open literature. Time resolved data was presented among others by (Dunn et al., 1984) and (Abhari et al., 1992) using thin film heat flux gauges. Endwall heat transfer measurements in a rotating facility were firstly presented by (Blair, 1992). Recently, (Lazzi Gazzini et al., 2017b) presented high-resolution heat transfer measurements using high-speed infrared thermography on the rotor endwall

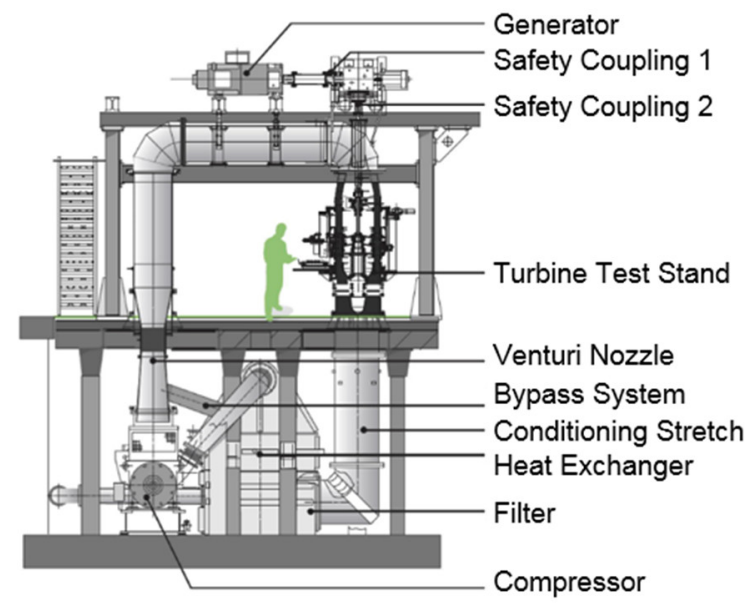

Figure 1: Overview of the turbine test facility "LISA" at ETH Zurich. from measurements in the same facility as used for this study. They found that the purge flow influences the Nusselt number distribution on the suction side of the rotor passage up to about $30 \%$ axial extent. However, the measured differences were within the measurement uncertainty.

Previous studies were focusing on endwall contouring within the rotor passage or purge flow injection separately. This work investigates an endwall design which is extending into the cavity and therefore strongly interacts with the purge flow. High resolution experimental data is presented from measurements in a rotating facility.

\section{METHODOLOGY}

\section{Research Facility and Operating Conditions}

The data presented in this paper is based on experiments in the axial turbine research facility "LISA" of the Laboratory for Energy Conversion (LEC) at ETH Zurich depicted in Figure 1. The test rig is a low-temperature, moderate-speed, continuously running rotating facility with non-dimensional parameter matching real engine conditions. A radial compressor is driving the air in the quasi-closed loop trough a two-stage water to air heat exchanger and a flow conditioning stretch before the test section. The turbine inlet total temperature is controlled with an accuracy of $\pm 0.4 \mathrm{~K}$. After the turbine section, the loop is open to atmosphere and the flow is fed back through a calibrated Venturi nozzle for mass flow measurements to the compressor. The turbine shaft is connected to a reduction gearbox and a DC generator breaking the turbine and controlling the rotational speed with an accuracy of $\pm 0.02 \%$ ( $\pm 0.5 \mathrm{rpm})$. The generated power is fed back to the power grid. Since the air loop is open to atmosphere, two rows of subsequent de-swirling vanes are installed to recover the static pressure back to ambient level. A more detailed description of the rig and test section is given by (Behr et al., 2006).

The investigated turbine configuration is a 1.5-stage, high-pressure turbine representative design. All blade rows and endwalls are 3D optimized geometries for reduced aerodynamic losses. The blade count for stator 1 , rotor and stator 2 are 36,42 and 36 respectively.

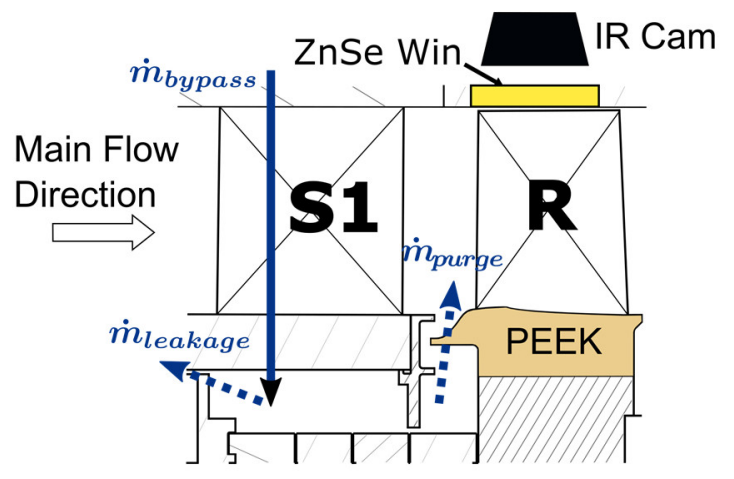

Figure 2: Schematic cut of first stage with purge flow injection path and optical access to the rotor endwall. 
Compressor air from the main loop is bypassed and used as cavity purge flow in the rotor upstream cavity. The air is extracted before the flow stretch and injected through 10 tubes in the first stator vanes into the cavity below. The purge flow injection rate $(I R)$ from the cavity into the main annulus is defined as:

$$
I R=\frac{\dot{m}_{\text {bypass }}-\dot{m}_{\text {leakage }}}{\dot{m}_{\text {tot }, \text { turbine }}} \cdot 100
$$

The purge air calculated from the mass flow balance of extracted bypass flow and cavity sealing leakage flow through the drum, normalized by the total turbine mass flow (Figure 2). Both mass flow rates are measured with calibrated standard nozzles.

The turbine was operated at the constant design pressure ratio of $\Pi_{1.5}=1.65$, accounting for changes in atmospheric pressure. Further relevant operating parameters are summarized in Table 1.

Table 1: Rig operating conditions.

\begin{tabular}{|c|c|c|}
\hline Parameter & Value & Units \\
\hline $\begin{array}{l}\text { Total rotor relative inlet } \\
\text { temperature } \boldsymbol{T}_{\boldsymbol{t}, \text { rel }}\end{array}$ & $313.5 \pm 0.1$ & {$[\mathrm{~K}]$} \\
\hline Pressure ratio $\Pi_{1.5}$ & $1.65 \pm 0.4 \%$ & {$[-]$} \\
\hline Rotor Inlet Mach number & 0.26 & {$[-]$} \\
\hline Mass flow & 11.7 & {$[\mathrm{~kg} / \mathrm{s}]$} \\
\hline Rotor speed & 2700 & [rpm] \\
\hline Purge Injection Ratio $\boldsymbol{I} \boldsymbol{R}$ & $0.0 \% / \mathbf{0 . 8} \% / 1.2 \%$ & {$[-]$} \\
\hline Purge cavity temperature $\boldsymbol{T}_{\boldsymbol{c}}$ & $326.4 / 325.4 / 324.2$ & {$[\mathrm{~K}]$} \\
\hline
\end{tabular}

\section{Rotor Endwall and Cavity Sealing Geometry}

The rotor upstream cavity sealing is an overlapping anglewing type of sealing with $2.1 \% c_{a x}$ of overlapping (Figure 3 ). Additionally, a radial sealing arm is separating the injection plenum from the rotor disk. This sealing geometry is representative to real engines designs.

The non-axisymmetric rotor hub endwall contouring is extended upstream into the rotor sealing arm arrangement. The contouring starts at $-23 \% c_{a x}$ upstream the rotor leading edge, at a radial location inside the sealing arrangement below the nominal hub radius of $330 \mathrm{~mm}$. The contouring consists of a pronounced hill on the pressure side of the passage next to a trough at the suction side at around the blade leading edge axial location (Figure 3, right). As shown, most of the contouring is located upstream of the leading edge until $50 \%$ axial chord. The rotor blades have a constant fillet radius of 3 $\mathrm{mm}$.

\section{Design Intention of Rotor Endwall}

The design of the rotor endwall contouring is based on a CFD optimization cycle which uses only the 1.5-stage aerodynamic efficiency as optimization target with mass flow as constrain. The steps were the following:

(1) The geometry is created using CAESES by Friendship Systems. The design of the non-axisymmetric endwall required only four parameters. Hill and trough are

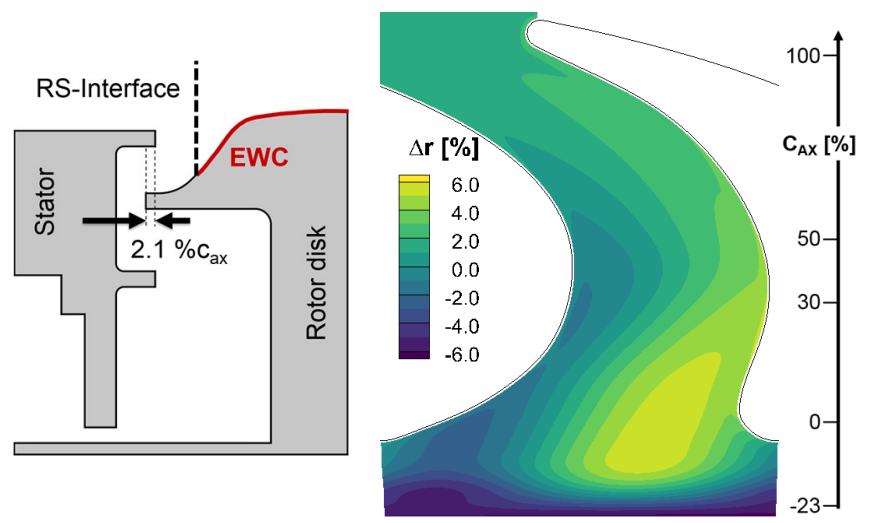

Figure 3: Rotor stator hub cavity sealing configuration (left) and hub endwall contouring (right).

both specified in terms of amplitude, axial position, axial extent and circumferential propagation relative to the camber line of the airfoil. The endwall has been optimized together with the airfoil by adding these additional parameters to the converged optimization setup of the blade.

(2) A block-structured mesh is generated with AutoGrid by Numeca. The domain includes three blade rows as well as the rotor upstream cavity. Only the rotor blade row is remeshed during the design optimization to save computational cost. A butterfly topology is used to generate a fillet radius between the airfoils and the non-axisymmetric endwall. The main gas path is modified such that it dips into the cavity for two reasons: (a) The radius at the platform leading edge can be modelled correctly and (b) the non-conformal interface between cavity and rotor blade (RS-Interface) is shifted upstream allowing for an extension of the endwall contouring (EWC) into the cavity (Figure 3, left).

(3) The steady CFD simulations are conducted using TRACE, a code specifically developed for turbomachinery applications by the Institute of Propulsion Technology at DLR. Radial distributions of total quantities and static pressure from previous measurements serve as inlet and outlet boundary conditions. The purge air is specified by a mass flow boundary condition of $0.8 \%$ of the main flow and turbulence is modelled with the shear stress transport model (SST).

This toolchain is run with AutoOpti by DLR, using a generic algorithm for the generation of designs and a surrogate model based on kriging. In total 89 design parameters were used for airfoil and endwall. The optimization required approximately 1500 members to converge. More details about the optimization algorithm are given in (Aulich and Siller, 2011).

A difference in efficiency of 0.3 percentage points in 1.5 stage efficiency compared to flat endwall was achieved in the optimization. However, as the airfoil and endwall have been optimized in combination, it is difficult to isolate the effect of the EWC itself. Compared to a flat endwall, a more uniform mass distribution of the purge over the circumference was observed. Also, the cross-passage migration of the pressure side horse shoe leg is shifted downstream and the passage vortex and suction side horse shoe vortex leg remain separated. This leads to two small pressure loss cores compared to one big loss core without contouring. 


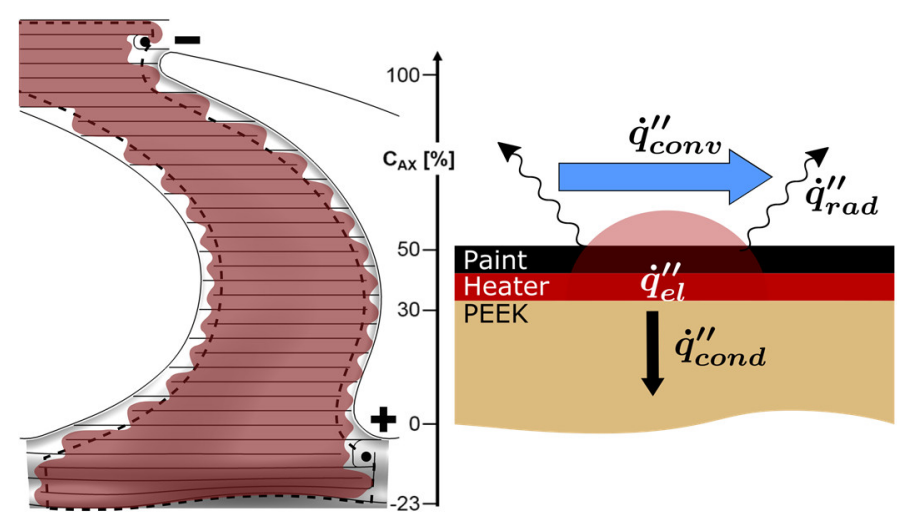

Figure 4: Endwall heating track design with indicated heated area (left) and energy balance of the setup (right).

\section{Experimental Setup}

Heat transfer measurements in the facility are performed with an infrared based technique described by (Lazzi Gazzini et al., 2017b) and (Laveau et al., 2014). A Flir IR SC7300L camera is used to record temperature of the rotor endwall through infrared transparent Zinc-Selenide (ZnSe) windows installed in the rotor casing (Figure 1). The camera is mounted on a traversing system, allowing vertical adjustments and different camera angles using various angle shims to extend the optical coverage through the window.

A quasi iso-energetic setup is used to create controlled boundary conditions at the region of interest. Therefore, a custom made thin film surface heating insert was manufactured and installed on the rotor. The insert is made out of PEEK (Polyether-ether-ketone), a thermal low conductive, high strength base substrate, replicating the exact endwall geometry. Pt100 temperature sensors are installed in the insert $1.5 \mathrm{~mm}$ below the surface to account for conduction losses. The surface is coated with an $1.4 \mu \mathrm{m}$ thick layer of Nickel using a direct chemical deposition technique on PEEK. Pulsed laser ablation is used to create a serpentine like heating track with a uniform width of $1.9 \mathrm{~mm}$. The heating track on the endwall is depicted in Figure 4, indicating also the achieved heated area in red. The inherent need for track turns results in unheated areas close to the blade fillet which are therefore excluded in the post-processing. For better infrared signature and reduction of reflections, all rotor surfaces are painted with Nextel black velvet high emissivity paint ( $\varepsilon=0.98$ (Kwor and Matteï, 2001)).

Nonuniformities in heat flux creation are corrected in the post-processing with the non-uniformity factor $S_{q}$ derived from transient heating tests, as described by (Lazzi Gazzini et al., 2017a) and defined in Equation (2). This factor accounts for local differences in electrical resistance or current concentrations in track turning points.

$$
S_{q}=\frac{\dot{q}_{e l, l o c}^{\prime \prime}}{\dot{q}_{e l, a v g}^{\prime \prime}}
$$

The temperature sensor data acquisition and control of the heaters are done with a rotating data acquisition system mounted on the rotor disk controlled over a wireless connection (Mansour et al., 2015). The electrical power for the heating platforms as well as for the rotor on-board data acquisition system is transferred to the rotating system with a two channel slip-ring. The heater supply current is measured and recorded externally.

\section{Image Processing}

Measurements are performed for a specific stator 1 to rotor relative position. A shaft mounted optical triggering system is used to trigger the infrared camera acquisitions. Images are recorded with a sensor integration time of $10 \mu$ s to reduce the blurring due to the platform movement. For extended optical coverage of the endwall, three different camera tilting angles $\left(-4.5^{\circ},+4.5^{\circ}\right.$ and $\left.+15^{\circ}\right)$ are used. For each position, a sequence of 1000 individually triggered images is recorded. All frames of an image sequence are registered with respect to a reference and only images within $\mathrm{a} \pm 1$ pixel shift are considered for the post-processing. Three different platform power levels with an averaged produced heat flux between $3.3 \mathrm{~kW} / \mathrm{m}^{2}$ to $9.2 \mathrm{~kW} / \mathrm{m}^{2}$ are used for the calculation of heat transfer quantities.

Silver infrared reference markers on the endwall are used to project the two dimensional image data on the three dimensional endwall geometry by geometrical transformation.

\section{Heat Transfer Calculation}

The convective heat flux $\dot{q}_{c o n v}^{\prime \prime}$ is calculated from the overall energy balance (Eqn. 3) on the heater surface, schematically depicted in Figure 4.

$$
\dot{q}_{c o n v}^{\prime \prime}=\dot{q}_{e l}^{\prime \prime}-\dot{q}_{c o n d}^{\prime \prime}-\dot{q}_{r a d}^{\prime \prime}
$$

The electric heat flux $\dot{q}_{e l}^{\prime \prime}$ is produced by Joule heating (eq. (4)) and directly calculated from the supply voltage and current and corrected for local non-uniformity by the factor $\mathrm{S}_{\mathrm{q}}$. The radiative heat flux $\dot{q}_{\text {rad }}^{\prime \prime}$ is calculated according to equation (5) with the emissivity $\varepsilon$ of the paint and $\sigma$ being the Stefan-Boltzmann constant. The conduction losses $\dot{q}_{\text {cond }}^{\prime \prime}$ are calculated using the installed temperature sensors inside the heater, according to the one dimensional conduction equation (6) based on the thermal properties of the substrate.

$$
\begin{gathered}
\dot{q}_{e l}^{\prime \prime}=S_{q} \frac{V \cdot I}{A_{\text {surf }}} \\
\dot{q}_{\text {rad }}^{\prime \prime}=\varepsilon \cdot \sigma \cdot\left(T_{w}^{4}-T_{t, r e l}^{4}\right) \\
\dot{q}_{\text {cond }}^{\prime \prime}=\frac{k_{\text {sub }}}{d_{\text {sub }}} \cdot\left(T_{w}-T_{\text {sub }}\right)
\end{gathered}
$$

The conduction losses are calculated for every heat flux step and averaged over the passage for the energy balance.

For each heating step, the convective heat flux and wall temperature are obtained. A least square linear fitting was used to calculate the local heat transfer coefficient $h$ and adiabatic wall temperature $T_{a w}$ as defined in equation (7) from the three heating steps.

$$
\dot{q}_{c o n v}^{\prime \prime}=h \cdot\left(T_{w}-T_{a w}\right)
$$




\section{Numerical Setup}

A multi-block structured mesh was generated for the 1.5 stage configuration including cavities. The mesh consisted of about 11.6 million nodes which provided a high-quality boundary layer resolution. The distribution of the nondimensional wall distance $y^{+}$for the simulation domain is shown in Figure 5.

The numerical investigation was performed with the commercial solver ANSYS CFX version 17.0. Steady state computations using the high-resolution advection scheme and the total energy equation including viscous work terms for compressible flow were conducted. Computations were performed with the SST turbulence model with the $\gamma-\theta$ transition model and mixing plane for the stator-rotor interface.

The surface roughness from the experiments was modelled using the roughness model supplied in CFX. Details about the model are described by (Lutum et al., 2015). Local roughness quantities were derived by profilometer measurements along the blade endwall. From this investigation an equivalent sand grain roughness level of $20 \mu \mathrm{m}$ was determined and applied for current predictions. The spatial discretization was quasi second order for all investigated cases.

Inlet profiles for total pressure, total temperature and turbulence quantities were obtained from corresponding experiments. The purge flow boundary conditions are specified by a mass flow rate of IR $=0.8 \%$ and total temperature of $328 \mathrm{~K}$. Numerical heat transfer results were determined from a set of computations consisting of an adiabatic and a heat flux calculation for the same operating conditions.

\section{RESULTS AND DISCUSSION}

Distributions of heat transfer quantities are shown for the three different purge flow injection ratios as defined in Table 1 . The presented results have been smoothed to reduce noise and clarify the data by using 30 passes of Tecplot 360 smoothing with a relaxation factor of 0.5 .

The local heat transfer coefficient is represented as Nusselt number as defined in Equation 8:

$$
N u=\frac{h \cdot c_{a x}}{k_{\text {air }}}
$$
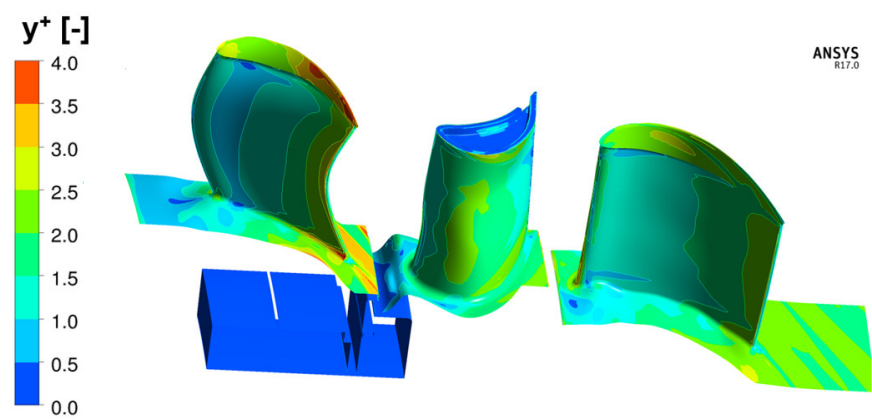

Figure 5: Non-dimensional wall distance for CFD simulations.
Where $c_{a x}$ is the rotor axial chord at mid-span and $k_{\text {air }}$ is the conductivity of air. The adiabatic wall temperature is presented for the design case in a non-dimensional form given in Equation (9).

$$
\theta=\frac{T_{a w}-T_{t, r e l}}{\frac{w^{2}}{2 c_{p}}}
$$

All flow reference quantities are mid-span circumferentially averaged values at the rotor inlet. Values are taken from experimental probe measurements with the LEC in-house manufactured fast response aerodynamic probe (FRAP) performed during the same measurement campaign. More details on the FRAP probe and measurement techniques can be found in (Kupferschmied et al., 2000) and (Pfau et al., 2003).

\section{Measurement Uncertainty}

The measurement uncertainty evaluation is performed by successively perturbing the input parameters in the postprocessing routine as described by (Moffat, 1988) as sequential perturbation. The local distribution of Nusselt number and non-dimensional adiabatic wall temperature uncertainty for the design case are presented in Figure 6.

For the measurements presented, the platform averaged relative uncertainty is reported to be $\pm 12.0 \%$ for the Nusselt number and absolute uncertainty of \pm 0.14 [-] for the nondimensional adiabatic wall temperature. The main contributors for the Nusselt number uncertainty are the conductive losses $(67.9 \%)$, the surface temperature $(27.2 \%)$ and non-uniformity scaling factor $S_{q}(3.5 \%)$. For the adiabatic wall temperature, the uncertainty in surface temperature $(70.3 \%)$ and conduction losses $(29.7 \%)$ are the main contributors. The uncertainty contributions are summarized in Table 2.

\section{Table 2: Relative measurement uncertainty} contributions.

\begin{tabular}{lrrrr}
\hline & $\delta T_{w}$ & $\delta \dot{q}_{\text {cond }}^{\prime \prime}$ & \multicolumn{1}{l}{$\delta S_{q}$} & \multicolumn{1}{l}{$\delta I$} \\
\hline$\delta N u$ & $27.2 \%$ & $67.8 \%$ & $3.5 \%$ & $1.5 \%$ \\
$\delta \theta$ & $70.2 \%$ & $29.7 \%$ & $0.0 \%$ & $0.0 \%$ \\
\hline
\end{tabular}

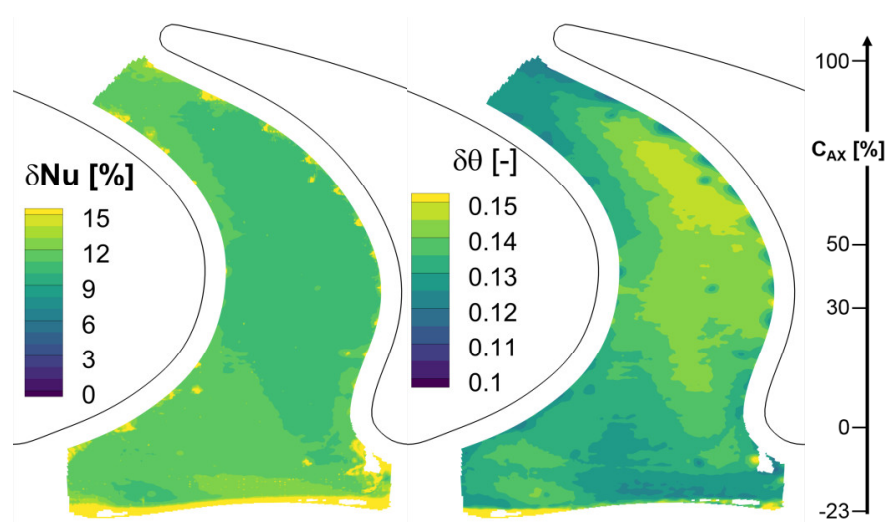

Figure 6: Distribution of relative Nusselt uncertainty (left) and absolute adiabatic wall temperature uncertainty (right). 

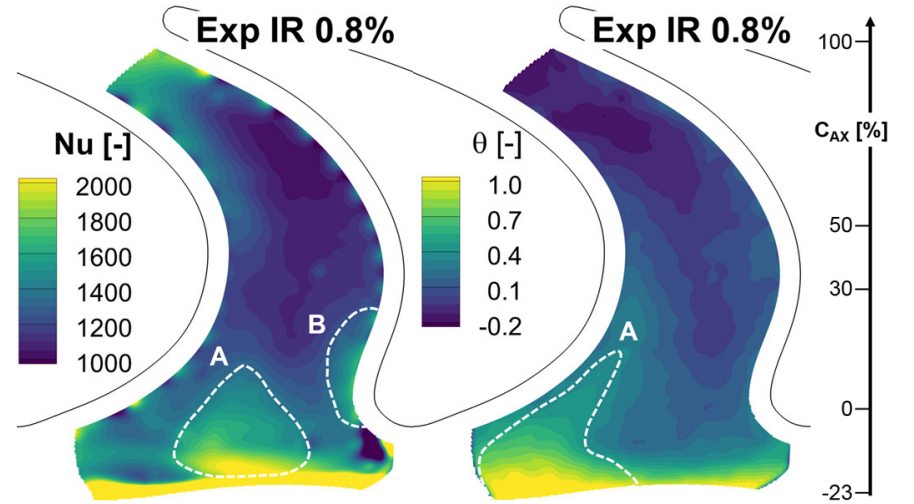

Figure 7: Nusselt number (left) and adiabatic wall temperature (right) distributions for design purge flow rate (IR 0.8\%).

\section{Endwall Heat Transfer for Design Point Operation}

Figure 7 shows the Nusselt and adiabatic wall temperature distribution on the hub endwall for the design purge condition $I R=0.8 \%$.

The small band of very high heat transfer at the beginning of the heated platform is consistent with results reported previously with a similar measurement setup ((Lazzi Gazzini et al., 2017b) and (Laveau et al., 2013)) and arises from the start of the thermal boundary layer. A wedge like region of increased heat transfer is following at mid-passage position and extending to about $10 \% c_{a x}$ from leading edge measured at mid-span (region A). This region coincides with the hill of the endwall contouring (Figure 3 ) and can be explained by the induced acceleration of the flow. Whereas towards the leading edge suction side of the blade lower heat transfer is observed following the endwall trough. The isolated small region upstream the leading edge with very low heat transfer is introduced by the electrical connection of the thin film heater and neglected in the analysis. An increase in heat transfer is observed towards the throat region and attributed to the flow acceleration.

The adiabatic wall temperature (Figure 7 , right) is decreasing through the rotor passage. This is expected as the flow temperature is decreasing due to expansion and acceleration. As the bypass air is extracted upstream of the turbine, the injected purge flow has higher temperature than the main flow. Therefore, contrary to cooling in real gas turbines, the purge flow is heating the endwall in this measurement setup. The very start of the platform shows the highest adiabatic wall temperature over the full pitch with isolines following closely the shape of the endwall contouring (Figure 3).

As this part of the endwall is extending inside the cavity and below the hub radius of the upstream NGV, it can be concluded that the changes seen in this region are associated to the interaction of purge flow. The extent of increased adiabatic wall temperature at the suction side up to $30 \%$ axial chord indicates that a high amount of hot purge air is entrained into the suction side horse shoe vortex, whereas the rapid decrease over the endwall hill indicates the presence of main flow.

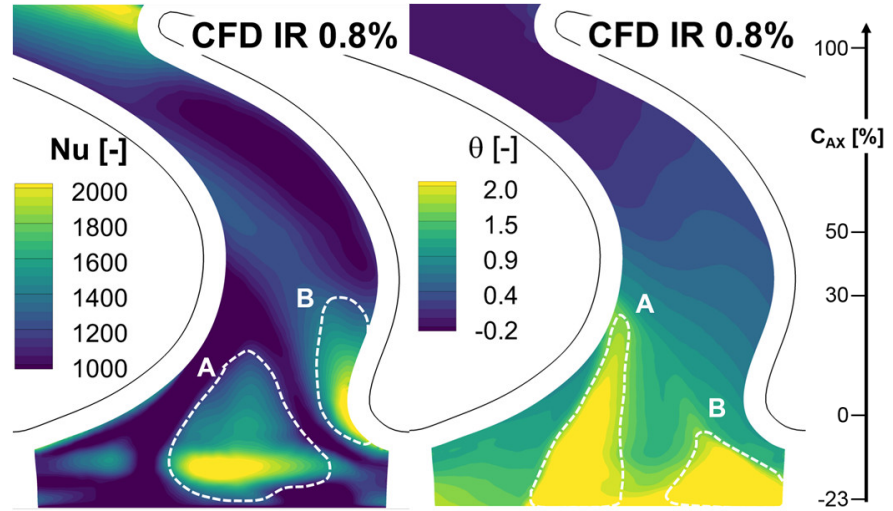

Figure 8: Distributions form CFD simulations of Nusselt number (left) and adiabatic wall temperature (right).

\section{Comparison with CFD predictions}

The predictions from CFD simulations are compared to the experimental data by means of endwall resolved Nusselt number and non-dimensional adiabatic wall temperature distribution and lateral averaged data of these values.

The simulation results for design purge flow rate are depicted in Figure 8. The increase of local heat transfer at the endwall hill upstream of the leading edge is clearly captured by CFD and visible as a wedge-like region (region A). The effect of the horse shoe vortex at the pressure side leading edge (region B) is not as clearly visible in the experiments as in CFD, however in both cases a local increase of heat transfer is observed. No influence of the horse shoe vortex on the suction side is visible for both the experiments and simulation as the effect is too close to the blade and therefore not covered by the heated surface of the measurement setup. The adiabatic wall temperature shows two locations of purge entrainment into the passage with increased temperature (region A and B). The larger region following the side of the endwall extending up to $30 \% c_{a x}$ is similar to the hot streak in the experiments (Figure 7, right) except for a slight pitch-wise shift. The second region (B) is not visible in the experiments. The relevant patterns for both quantities are captured in the simulation.
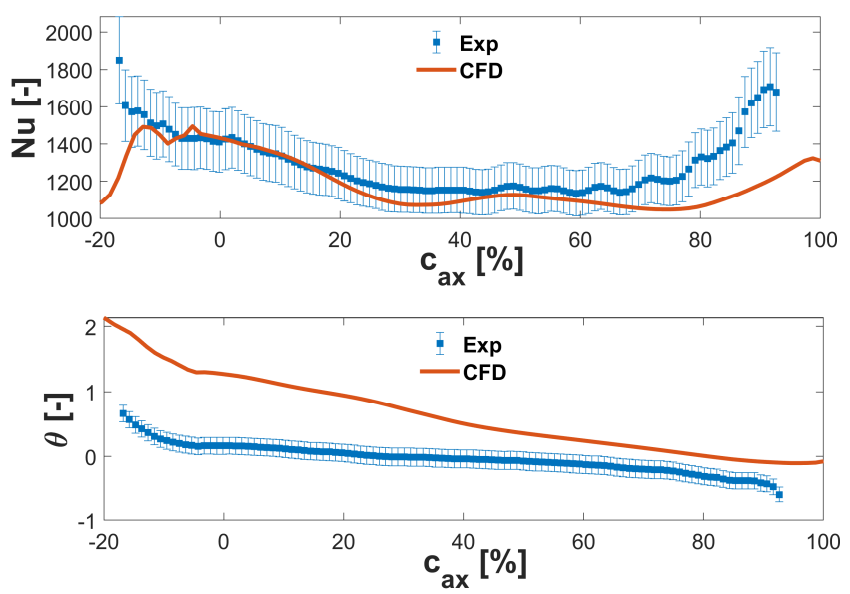

Figure 9: Lateral averaged Nusselt number (top) and adiabatic wall temperature (bottom) for CFD and Experiments. 
The lateral averaged results are compared in Figure 9. A general agreement within $\pm 15 \%$ for Nusselt number is found. The high heat transfer region at the start of the heater is not captured by the simulations. Also, the increase through the passage is predicted less pronounced and to start farther downstream than measured in the experiment. The trends for the adiabatic wall temperature are captured with a change in slope at around $-5 \% c_{a x}$. The offset in adiabatic wall temperature in the simulation, especially at the beginning of the platform, is explained by a miss-match in purge flow temperature and a different heat pickup compared to the experiments.

\section{Purge Flow Influence on Endwall Heat Transfer}

Effect of purge flow on the endwall heat transfer is investigated with three different injection rates. For that, the design point $I R=0.8 \%$ case is the reference and compared against zero injection $I R=0.0 \%$ and a high injection rate $I R=1.2 \%$. This is done by means of the relative Nusselt number difference defined as:

$$
\Delta N u=\frac{N u_{I R}-N u_{I R 0.8 \%}}{N u_{I R 0.8 \%}}
$$

Figure 10 shows the relative difference to nominal injection for both off-design cases. A general observation reveals, for both cases, local changes in heat transfer up to $\pm 20 \%$. However, significant changes are only found within the first $30 \%$ of axial chord length. This implies that after the passage cross flow hits the blade suction side, all the purge flow is mixed into the secondary flows and detached from the endwall.

In the case of zero purge injection, a distinct increase of heat transfer is observed along the suction side from the leading edge downstream to about $c_{a x}=0.3$. This implies, that the purge flow in the reference case interacts with the suction side horse shoe vortex which usually enhances the local heat transfer due to increased shear. However, for the investigated geometry with endwall contouring extending into the cavity, the purge flow leaves the cavity close to the blade suction side as shown in Figure 11 (left) by CFD. The low momentum purge flow is reducing the local flow velocity and therefore the heat transfer. Over the endwall hill region, a slight decrease of heat transfer is observed for no purge flow.

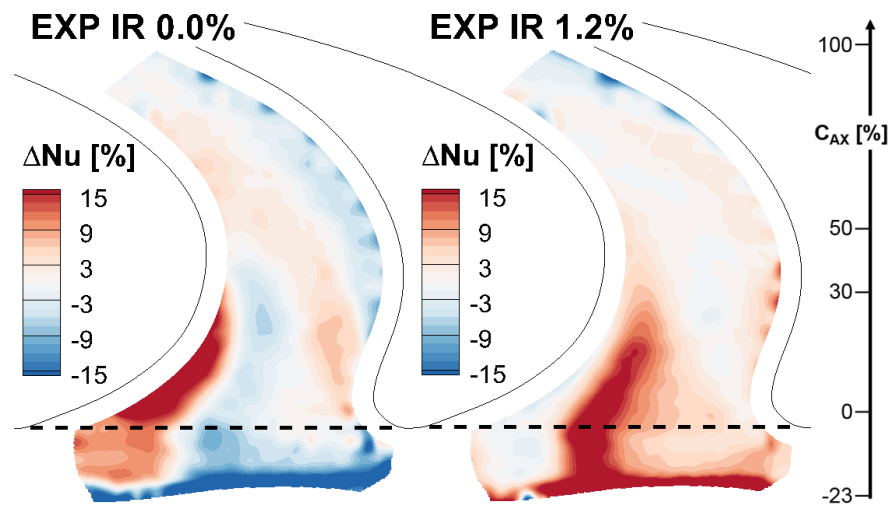

Figure 10: Relative difference in local Nusselt number for IR $=\mathbf{0 . 0} \%$ (left) and IR $1.2 \%$ (right) compared to design point (IR $0.8 \%$ ).

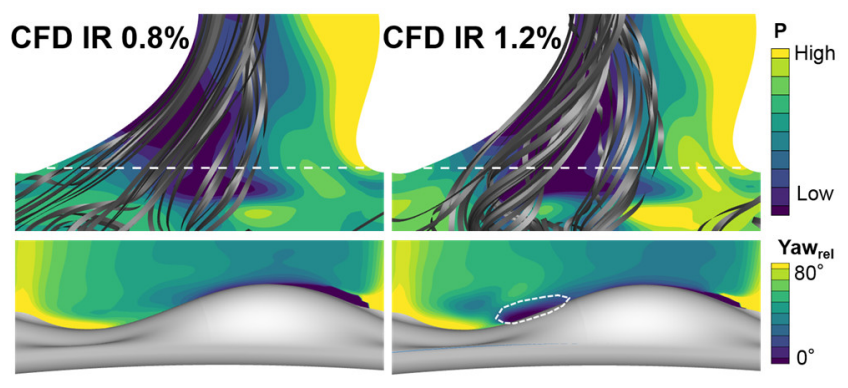

Figure 11: Purge flow stream lines (top) and relative yaw angle contour (bottom) for IR $0.8 \%$ (left) and IR $1.2 \%$ (right).

Additionally, the trace of increased heat transfer starting from the pressure side leading edge over the endwall to about $50 \%$ $c_{a x}$ indicates a stronger influence of the horse shoe vortex migration for the case without purge flow injection.

For the case of high purge flow rate, increased heat transfer is observed in a triangular region extended up to $35 \%$ axial chord where it ends at the blade suction side. The highest increase of up to $20 \%$ is located in a band like region starting from the platform start along the side of the endwall hill.

For $I R=1.2 \%$, the purge flow is entrained as two different jets into the main flow and endwall (Figure 11). Due to the higher purge momentum, the relative yaw angle of the main flow is reduced by the purge and enhanced mixing of both flows is present. Additionally, the purge entrainment at the suction side causes a mass redistribution and flow acceleration of the main flow in the region between the two purge jets.

The influence of purge rate on the pitch wise distribution of absolute heat transfer over the passage is shown in Figure 12, for the indicated dashed line in Figure 10. Towards the suction side, both purge flow rates of $0.8 \%$ and $1.2 \%$ show nearly identical levels of heat transfer, where the zero purge rate shows the same trend but with elevated levels. This reduction in heat transfer is caused by the local reduction in velocity due to the presence of low momentum purge air as described before. In-between 0.3 and 0.6 rotor pitch, the heat transfer is highest modulated due to purge flow. Without purge flow the most uniform circumferential heat transfer distribution on the endwall is achieved. The modulation due to purge flow is similar for both injection ratios, $0.8 \%$ and $1.2 \%$, with different amplitudes at the maximum level leading to an increased non-uniformity over the pitch.

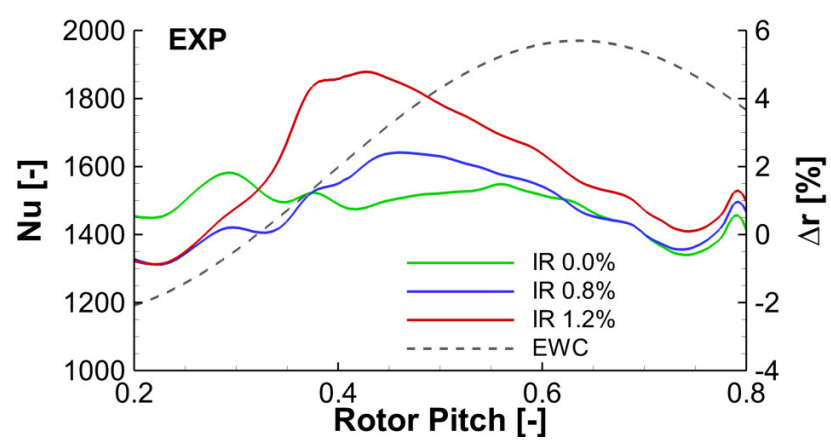

Figure 12: Pitch-wise Nusselt number distribution and endwall radius change at indicated location in Figure 10. 


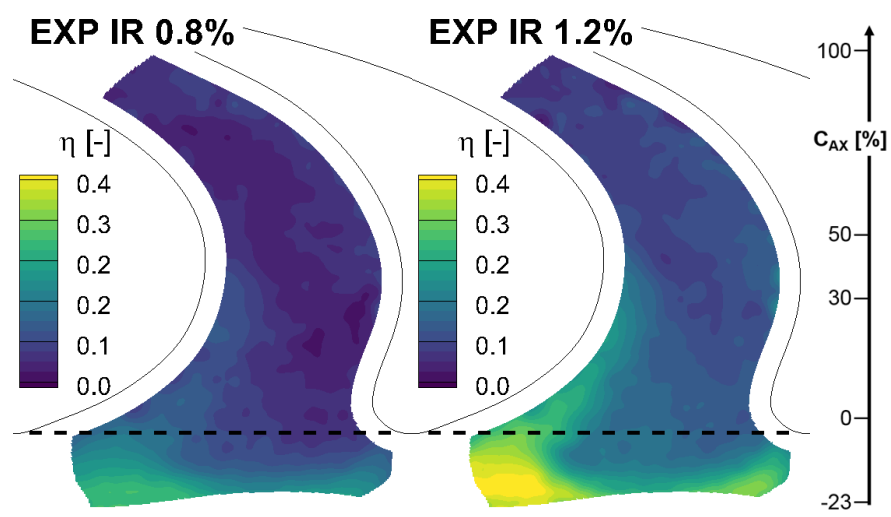

Figure 13: Experimental endwall cooling effectiveness for IR $0.8 \%$ (left) and IR $1.2 \%$ (right).

\section{Purge Flow Endwall Cooling Effectiveness}

The cavity purge flow injection not only affects the main flow and secondary flow structures in the rotor passage, but also provides cooling of the endwall due to the temperature difference to the main flow. This cooling is evaluated by means of a cooling effectiveness $\eta$, as defined in Equation (11), where the adiabatic wall temperature of the zero purge flow case is taken as a reference.

$$
\eta=\frac{T_{a w}-T_{a w, I R 0.0 \%}}{T_{c}-T_{t, r e l}}
$$

The difference in adiabatic wall temperature is normalized by the difference of the purge flow temperature $T_{c}$ measured in the cavity upstream the radial sealing arm and the rotor inlet relative total temperature.

Figure 13 shows the cooling effectiveness for nominal purge rate and high purge rate for the rotor endwall. The general pattern is the same for both cases. The highest cooling effect is observed at the very beginning of the platform, where the endwall is below the nominal main flow hub radius and therefore immersed in the purge flow. The other area of purge cooling is starting at the EWC trough and extending up to $30 \%$ axial chord along the blade suction side. The pattern is very similar to the regions of decreased heat transfer due to purge flow injection discussed in the previous section (Figure 9). This further confirms that the purge flow is, in a large part, swept to the suction side of the blade. This concentration is also illustrated in Figure 14, which shows the circumferential distribution for the same axial position indicated in Figure 13.

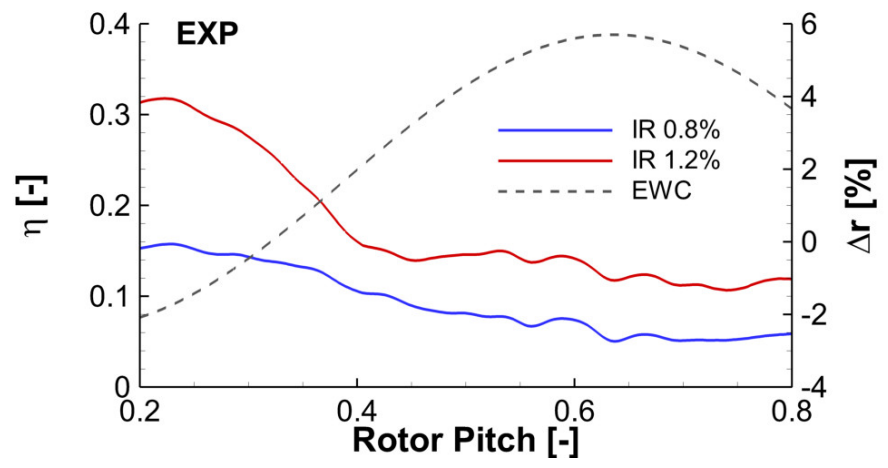

Figure 14: Pitch wise cooling effectiveness distribution at indicated location in Figure 13.
The effectiveness is about twice as high at the suction side with the endwall trough compared to the rest of the pitch.

The cooling effectiveness is about $75 \%$ higher for the high injection case, over the design injection, where the overall platform averaged effectiveness is 0.085 for $\mathrm{IR}=0.8 \%$ and 0.150 for $\mathrm{IR}=1.2 \%$.

\section{CONCLUSIONS}

This work presents high-resolution heat transfer measurement data of a high-pressure representative turbine rotor endwall with advanced non-axisymmetric endwall contouring in a rotating turbine facility. A new technique was used to manufacture a thin film quasi-iso-energetic heating surface on arbitrarily shaped surfaces and to create controlled thermal boundary conditions for fast infrared surface temperature measurements. The influence of different purge flow injection rates on endwall heat transfer and cooling effectiveness was investigated for a contoured endwall extended into the disk cavity.

Predictions from CFD simulations generally showed a good agreement with the experiments within $\pm 15 \%$ and capture the relevant local heat transfer patterns.

Cavity extended non-axisymmetric endwall contouring is shown to significantly influence the local heat transfer rate from the beginning of the platform up to $30 \%$ axial blade chord. Differences of up to $20 \%$ in local Nusselt number were observed for no purge flow and high purge flow rate compared to design conditions. Endwall resolved CFD results are required to resolve these local changes, which are not captured by lateral averaged data.

An average cooling effectiveness of 0.085 and 0.150 for purge rates of $0.8 \%$ and $1.2 \%$ respectively are reported compared to the case without purge flow.

Therefore, the results suggest that during the design and optimization process of advanced geometries for highest aerodynamic efficiencies, auxiliary air injection should be included, as well as the local heat load.

Advanced tools for aerodynamic performance optimization can lead to very specific designs. For the investigated geometry, the injection of purge flow is beneficially reducing the heat transfer at the stress critical region of the blade root leading edge suction side. This benefit, however, is counteracted by increased pitch-wise differences and non-uniformities over the platform. Due to this sensitivity of heat transfer to the purge rate, also off-design performance needs to be considered during the design phase.

\section{NOMENCLATURE}

\section{Variables:}

c blade chord at mid-span $\quad[-]$

$h \quad$ heat transfer coefficient $\quad\left[\mathrm{W} / \mathrm{m}^{2} / \mathrm{K}\right]$

I heating current [A]

$k \quad$ thermal conductivity

$[\mathrm{W} / \mathrm{m} / \mathrm{K}]$

$\mathrm{Nu} \quad$ Nusselt number [-]

$S_{q} \quad$ non-uniformity heat flux scaling factor [-]

$T$ temperature [K]

$w \quad$ relative velocity $[\mathrm{m} / \mathrm{s}]$ 


$$
y^{+} \quad \text { dimensionless wall distance }
$$

\section{Greek}

$\varepsilon$

surface emissivity

$[-]$

non-dimensional adiabatic wall [-]

temperature

$\eta \quad$ cooling effectiveness

$[-]$

П $\quad$ pressure ratio

\section{Subscripts:}

$\begin{array}{ll}\text { aw } & \text { adiabatic wall } \\ \text { ax } & \text { axial direction } \\ \text { c } & \text { coolant/purge } \\ \text { cond } & \text { conductive heat flux } \\ \text { conv } & \text { convective heat flux } \\ \text { el } & \text { Joule electric heat flux } \\ \text { rel } & \text { relative frame of reference } \\ \text { rad } & \text { radiative heat flux } \\ \text { sub } & \text { substrate } \\ \mathrm{t} & \text { stagnation flow quantity } \\ \mathrm{W} & \text { wall }\end{array}$

\section{Abbreviations:}

CFD Computational Fluid Dynamics/Simulation

DLR Deutsches Zentrum für Luft- und Raumfahrt

EWC endwall contouring

EXP Experimental

FRAP fast response aerodynamic probe

IR injection rate

LEC Laboratory for Energy Conversion

NGV nozzle guide vane/ stator 1

RS Rotor-stator

SST shear stress transport model

ZnSe Zinc Selenide

\section{ACKNOWLEDGMENTS}

This work has been funded by the Federal Ministry for Economic Affairs and Energy via the funding programme Luftfahrtforschungsprogramm V, MTU Aero Engines AG and Siemens AG. The authors greatly acknowledge the financial support and the permission to publish. The responsibility for the content of the publication rests with the authors.

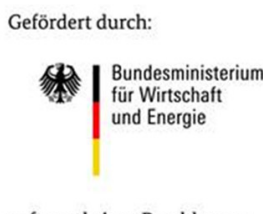

aufgrund eines Beschlusses
des Deutschen Bundestages

\section{REFERENCES}

ABHARI, R. S., GUENETTE, G. R., EPSTEIN, A. H. \& GILES, M. B. 1992. Comparison of Time-Resolved Turbine Rotor Blade Heat Transfer Measurements and Numerical Calculations. Journal of Turbomachinery, 114, 818-827.

AULICH, M. \& SILLER, U. 2011. High-Dimensional Constrained Multiobjective Optimization of a Fan Stage. 1185-1196.

BEHR, T., KALFAS, A. I. \& ABHARI, R. S. 2006. Unsteady Flow Physics and Performance of a One-and-1/2-Stage Unshrouded High Work Turbine. Journal of Turbomachinery, 129, 348-359.

BLAIR, M. F. An Experimental Study of Heat Transfer in a Large-Scale Turbine Rotor Passage. Turbo Expo: Power for Land, Sea, and Air, 1992. Volume 4: Heat Transfer; Electric Power; Industrial and Cogeneration, V004T09A012.

DUNN, M. G., RAE, W. J. \& HOLT, J. L. 1984. Measurement and Analyses of Heat Flux Data in a Turbine Stage: Part IDescription of Experimental Apparatus and Data Analysis. Journal of Engineering for Gas Turbines and Power, 106, 229-233.

HARTLAND, J. C., GREGORY-SMITH, D. G., HARVEY, N. W. \& ROSE, M. G. 1999. Non-Axisymmetric Turbine End Wall Design: Part II - Experimental Validation. V001T03A050.

HARVEY, N. W., ROSE, M. G., TAYLOR, M. D., SHAHPAR, S., HARTLAND, J. \& GREGORY-SMITH, D. G. 1999. Non-Axisymmetric Turbine End Wall Design: Part I - ThreeDimensional Linear Design System. V001T03A049.

JENNY, P., ABHARI, R. S., ROSE, M. G., BRETTSCHNEIDER, M., ENGEL, K. \& GIER, J. 2013. Unsteady Rotor Hub Passage Vortex Behavior in the Presence of Purge Flow in an Axial Low Pressure Turbine. Journal of Turbomachinery, 135, 051022-051022-9.

KUPFERSCHMIED, P., KÖPPEL, P., GIZZI, W., RODUNER, C. \& GYARMATHY, G. 2000. Time-resolved flow measurements with fast-response aerodynamic probes in turbomachines. Measurement Science and Technology, 11, 1036.

KWOR, E. T. \& MATTEÏ, S. 2001. Emissivity measurements for Nextel Velvet Coating 811-21 between -36 degrees C and 82 degrees C. 15th European Conference on Thermophysical Properties.

LANGSTON, L. S. 2001. Secondary Flows in Axial Turbines-A Review. Annals of the New York Academy of Sciences, 934, 11-26.

LAVEAU, B., ABHARI, R. S., CRAWFORD, M. E. \& LUTUM, E. 2013. High Resolution Heat Transfer Measurement on Flat and Contoured Endwalls in a Linear Cascade. Journal of Turbomachinery, 135.

LAVEAU, B., ABHARI, R. S., CRAWFORD, M. E. \& LUTUM, E. 2014. High Resolution Heat Transfer Measurements on the Stator Endwall of an Axial Turbine. Journal of Turbomachinery, 137.

LAZZI GAZZINI, S., SCHÄDLER, R., KALFAS, A. I. \& ABHARI, R. S. 2017a. Infrared thermography with non-uniform heat flux boundary conditions on the rotor endwall of an axial turbine. Measurement Science and Technology, 28.

LAZZI GAZZINI, S., SCHÄDLER, R., KALFAS, A. I., ABHARI, R. S., HOHENSTEIN, S., SCHMID, G. \& LUTUM, E. 2017b. Effect of purge air on rotor endwall heat transfer of an axial turbine. Journal of the Global Power and Propulsion Society, 1, 211223.

LUTUM, E., COTTIER, F., CRAWFORD, M. E., LAVEAU, B. \& ABHARI, R. S. 2015. A computational investigation of the effect of surface roughness on heat transfer on the stator endwall of an axial turbine. Proceedings of the Institution of Mechanical Engineers, Part A: Journal of Power and Energy, 229, 454-464.

LYNCH, S. P., SUNDARAM, N., THOLE, K. A., KOHLI, A. \& LEHANE, C. 2010. Heat Transfer for a Turbine Blade With Nonaxisymmetric Endwall Contouring. Journal of Turbomachinery, 133, 011019-011019-9. 
MANSOUR, M., REBHOLZ, P. S., KALFAS, A. I. \& ABHARI, R. S. An On-Board Wireless Multi-Sensor Measurement System for Rotating Turbomachinery Application. Proceedings of International Gas Turbine Congress 2015 Tokyo November 15-20, 2015, Tokyo, Japan, 20152015.

MOFFAT, R. J. 1988. Describing the uncertainties in experimental results. Experimental Thermal and Fluid Science, 1, 317.

PFAU, A., SCHLIENGER, J., KALFAS, A. I. \& ABHARI, R. S. 2003. Unsteady, 3-Dimensional Flow Measurement Using a Miniature Virtual 4 Sensor Fast Response Aerodynamic Probe (FRAP). 307-315.

REGINA, K., KALFAS, A. I. \& ABHARI, R. S. 2014. Experimental Investigation of Purge Flow Effects on a High Pressure Turbine Stage. Journal of Turbomachinery, 137, 041006-041006-8.

ROY, A., JAIN, S., EKKAD, S. V., NG, W., LOHAUS, A. S., CRAWFORD, M. E. \& ABRAHAM, S. 2017. Heat Transfer Performance of a Transonic Turbine Blade Passage in the Presence of Leakage Flow Through Upstream Slot and Mateface Gap With Endwall Contouring. Journal of Turbomachinery, 139, 121006121006-11.

SCHUEPBACH, P., ABHARI, R. S., ROSE, M. G., GERMAIN, T., RAAB, I. \& GIER, J. 2008. Improving Efficiency of a High Work Turbine Using Non-Axisymmetric Endwalls: Part IITime-Resolved Flow Physics. 1121-1133.

SCHUEPBACH, P., ABHARI, R. S., ROSE, M. G. \& GIER, J. 2010. Influence of Rim Seal Purge Flow on the Performance of an Endwall-Profiled Axial Turbine. Journal of Turbomachinery, 133, 021011-021011-10.

SHARMA, O. P. \& BUTLER, T. L. 1987. Predictions of Endwall Losses and Secondary Flows in Axial Flow Turbine Cascades. Journal of Turbomachinery, 109, 229-236.

SNEDDEN, G., DUNN, D., INGRAM, G. \& GREGORYSMITH, D. 2009. The Application of Non-Axisymmetric Endwall Contouring in a Single Stage, Rotating Turbine. 831-840.

THRIFT, A. A., THOLE, K. A. \& HADA, S. 2012. Effects of Orientation and Position of the Combustor-Turbine Interface on the Cooling of a Vane Endwall. Journal of Turbomachinery, 134, 061019-061019-10. 